\title{
Antimonide quantum dots enable novel photonic devices
}

\author{
Naokatsu Yamamoto, Kouichi Akahane, \\ Shin-ichirou Gozu, Akio Ueta, and Masahiro Tsuchiya
}

Structures based on antimonide III-V semiconductor quantum dots can provide communications lasers on inexpensive substrates.

Long-wavelength (i.e., near-IR) light-emitting devices, especially 1.3 - and $1.55 \mu \mathrm{m}$ lasers, are needed for ubiquitous fiberoptic communication networks. To achieve low-cost, low-power consumption and high performance from such light sources, we would like to fabricate them on low-cost, high-quality semiconductor substrates such as gallium arsenide and silicon wafers. But the crystal lattice mismatch between these substrates and narrow-energy-bandgap semiconductors makes it difficult to obtain near-IR light-emitting materials using normal fabrication techniques.

Recently, some approaches for creating these luminescent materials have been proposed. As one method, the epitaxial growth of nitride-based semiconductors-such as GaInNAson GaAs substrates is being widely investigated. SiGe, silicon quantum dots (QDs), ${ }^{1}$ and III-V semiconductors bonded directly to $\mathrm{Si}$ are also being studied to find a photonics technology that allows us to fabricate light-emitting materials on $\mathrm{Si}$. However, obtaining optimized material characteristics for highintensity and near-IR luminescence with these material fabrication techniques is also difficult. To avoid these difficulties, we used nanostructured semiconductors, such as a quantum dots (QDs), to create near-IR luminescent material on GaAs and $\mathrm{Si}$ substrates.

Quantum dots have very interesting characteristics, ${ }^{2}$ including quantum confinement of carriers and high luminescent efficiency. In addition, QD structures can be grown without requiring lattice matching between the QDs and the substrate. Without this restriction, we are free to use antimonide-based III-V semiconductor materials, which have very narrow bandgaps. These materials were not used in the past because of the very large lattice mismatch (more than $10 \%$ ) between Sb-based materials and GaAs or Si. Therefore, we created Sb-based III-V semiconductor $\mathrm{QD}$ structures (i.e., the $\mathrm{Sb}$ atoms are included in

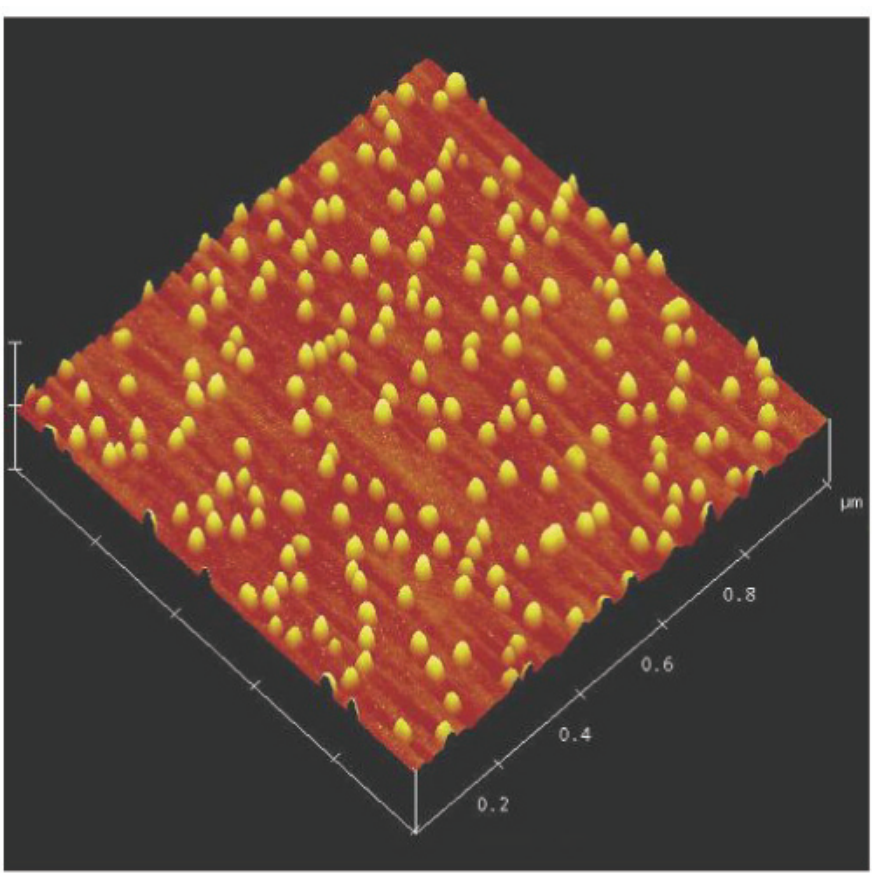

Figure 1. Atomic force microscope image of Sb-based quantum dots (QDs) on a GaAs surface.

or neighbor the QD structure) formed on GaAs and Si to achieve near-IR emission.

We grew the Sb-based QD structure using molecular beam epitaxy (MBE). First, we proposed a Si-atom irradiation technique and optimized the growth conditions to improve the density of the QDs (a density as high as $10^{10} / \mathrm{cm}^{2}$ is necessary to develop a laser or other light-emitting device). ${ }^{3,4}$ We believed that emission could be obtained by a confined-carrier recombination in this nanostructured $\mathrm{Sb}$-based semiconductor. Figure 1 shows the Sb-based QD structure formed on a GaAs surface under optimized growth conditions. The height and diameter of the QD structures are $\sim 7.5$ and $25 \mathrm{~nm}$, respectively. We also obtained a density as high as $2 \times 10^{10} \mathrm{QD} / \mathrm{cm}^{2}$. 


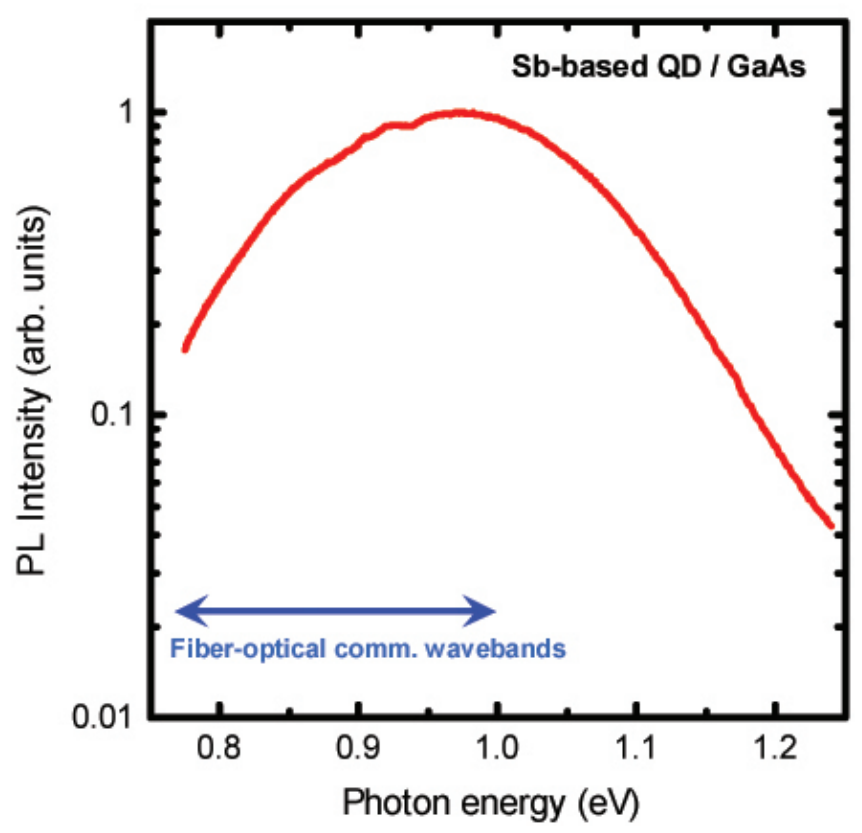

Figure 2. Light emission from Sb-based $Q D$ structures on GaAs in the fiber-optic communications waveband.

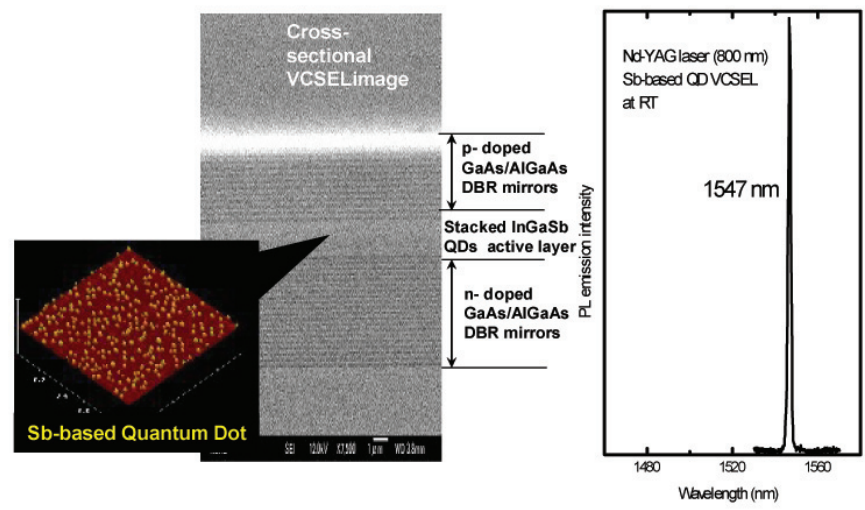

Figure 3. A Sb-based $Q D$ vertical-cavity surface-emitting laser (VCSEL) structure (left) and the emission spectrum from an optically pumped VCSEL structure (right).

We observed emission at wavelengths as long as 1.3- and $1.55 \mu \mathrm{m}$, as shown in Figure 2, from the Sb-based QD structure embedded in GaAs. We also obtained these wavelengths from an LED that contained QDs in the active regions. We tried to develop a vertical-cavity surface-emitting laser (VCSEL) containing Sb-based QDs because near-IR VCSELs are expected to be one of the candidates for the light source used in 10Gb Ethernet (or faster) optical networks. In addition, a high-performance distributed Bragg reflector (DBR) necessary to form the optical cavity in VCSELs is simple to form in the AlGaAs system.
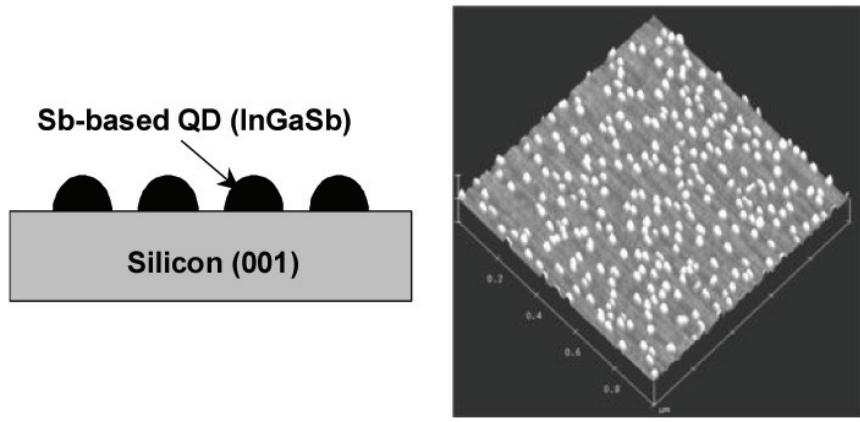

Figure 4. Sb-based III-V semiconductor QD structure on silicon.

We fabricated, respectively, current-injected and optically pumped Sb-QD VCSELs. Figure 3 presents a cross-section of our current-injection Sb-based QD VCSEL and the $1.55 \mu \mathrm{m}$ emission spectrum from the optically pumped VCSEL. The Sb-based QD active layers and AlGaAs DBR mirrors can be grown monolithically using epitaxial growth on a GaAs substrate. This is a simple way to make these VCSELs. We found that a $1.52 \mu \mathrm{m}$ emission peak can be obtained at room temperature with continuous current injection. ${ }^{4}$ A sharp $1.55 \mu \mathrm{m}$ emission peak and threshold characteristics of the curve of the optical pump power versus laser power were also observed from the optically pumped VCSELs. These results may indicate a possibility of laser operation in the $1.5 \mu \mathrm{m}$ waveband using the Sb-QD-VCSEL structure. However, we are continuing characterization and optimization of Sb-based QD materials and laser structures to clarify the lasing operation and develop the VCSEL for practical use.

Recently, we also focused on Sb-based QDs fabricated on a Si wafer for Si photonics technology (see Figure 4). We found that a high-density (more than $10^{10} / \mathrm{cm}^{2}$ ) of small (8nm) Sb-based QDs can be grown on Si using MBE. ${ }^{5}$ We expect that this will enable us to achieve energy-bandgap engineering, high carrier mobility, and near-IR light emission for novel photonic devices on $\mathrm{Si}$ wafers.

Previous techniques considered for forming light emitters at these wavelengths from semiconductor materials on GaAs and Si wafers were very difficult. To solve this problem, we proposed fabricating Sb-based QDs on these wafers, which emit in the low-loss window of optical fiber. We also successfully demonstrated $1.5 \mu \mathrm{m}$ emissions from a Sb-based QD VCSEL. Additionally, we fabricated a Sb-based QD structure on a Si wafer, which may become a novel material for making Si-light emitters and optoelectronics devices. We believe that Sb-based QDs on lowcost and high-performance GaAs and Si wafers will enable us to achieve breakthroughs in fabricating novel photonics devices for ubiquitous communication networks.

Continued on next page 
We thank H. Sotobayashi, T. Kawanishi, M. Izutsu, T. Itabe, and F. Tomita at NICT for encouragement throughout this work. We also thank Y. Mitsumori of Tohoku University, N. Ohtani of Doshisha University, and all of the staff at the Photonics Device Laboratory (PDL) of NICT for technical assistance and advice.

\section{Author Information}

\section{Naokatsu Yamamoto, Kouichi Akahane,}

Shin-ichirou Gozu, Akio Ueta, and Masahiro Tsuchiya

Advanced Communications Technology Group

National Institute of Information and Communications

Technology

Tokyo, Japan

http:/ /act.nict.go.jp/lwd/staff/naokatsu /

http:/ / act.nict.go.jp

Naokatsu Yamamoto is a researcher in the Advanced Communications Technology Group at NICT. His research interests include development and creation of novel photonic devices with attractive material characteristics and nanostructures.

\section{References}

1. N. Yamamoto, A. Sumiya, and H. Takai, Electroluminescence from photo-chemically etched silicon, Mater. Sci. Eng. B69-70, pp. 205-209, 2000.

2. Y. Arakawa and H. Sakaki, Multidimensional quantum well laser and temperature dependence of its threshold current, Appl. Phys. Lett. 40, pp. 939-941, 1982.

3. N. Yamamoto, K. Akahane, S. Gozu, and N. Ohtani, Over $1.3 \mu$ m continuous-wave laser emission from In GaSb quantum-dot laser diode fabricated on GaAs substrates, Appl. Phys. Lett. 86, p. 203118, 2005.

4. N. Yamamoto, K. Akahane, S. Gozu, A. Ueta, and N. Ohtani, 1.55- $\mu$ m-waveband emissions from Sb-based quantum-dot vertical-cavity surface-emitting laser structures fabricated on GaAs substrate, Jpn. J. Appl. Phys 45, pp. 3423-3426, 2006

5. N. Yamamoto, K. Akahanea, S. Gozu, A. Ueta, N. Ohtani, and M. Tsuchiya, Sbbased quantum dots for creating novel light-emitting devices for optical communications, Proc. SPIE 6393, 2006. Also Growth of In GaSb quantum dot structures on GaAs and silicon substrates, Jpn. J. Appl. Phys., submitted. 